\title{
GRAPHENE MICROTUBES - NEW TYPE OF CARBON MATERIALS
}

Evgeny V. Zaytsev, Elena S. Kurkina

Mendeleev University of Chemical Technology of Russia, https://muctr.ru

Moscow 125047, Russian Federation

Kirill A. Bukunov

Skobeltsyn Institute of Nuclear Physics Lomonosov Moscow State University, http://www.sinp.msu.ru Moscow 119234, Russian Federation

Grigory S. Bocharov, Alexander V. Eletskii

National Research University "Moscow Power Engineering Institute", http:/ / mpei.ru

Moscow 111250, Russian Federation

Pavel N. Chuprov, Sergey V. Tkachev, Denis Yu. Kornilov

LLC "АкKо Lab",

Moscow 129110, Russian Federation

Sergey P. Gubin

Kurnakov Institute of General and Inorganic Chemistry of the Russian Academy of Sciences, http://wwwigic.ras.ru Moscow 119991, Russian Federation

gliese3@gmail.com, bukunov@mail.ru, bocharov@mail.ru, chuprovp@rambler.ru, tkachev_ svmsu@mail.ru, kornilovdenis@rambler.ru, kurkina@mail.ru,gubin@igic.ras.ru, eletskii@mail.ru

Abstract. The possibility of obtaining a graphene coating on non-flat (cylindrical) surfaces, namely, copper fibers, is shown. Graphene coating is characterized by SEM and KR methods. The possibility of its removal by etching out the carrier substrate is demonstrated. Along with the growth of graphene, the formation of impurity particles on the surface of the copper fiber, which worsen the quality of graphene. The placement of fiber in a copper casing allowed to improve the quality of graphene coating on copper fibers and to suppress the formation of impurity structures on their surfaces.

Keywords: CVD reactor, copper fiber, graphene coating, impurity particles

UDC 541.1: 539.23

Bibliography - 11 references

Received May 05, 2018

RENSIT, 2018, 10(1):59-64

DOI: $10.17725 /$ rensit.2018.10.059

\section{Contents}

1. Introduction (59)

2. Materials and methods (60)

3. Results and discussion (61)

3.1. Impurity structures (61)

3.2. Growth of graphene on copper fiber (62)

4. Conclusion (62)

5. References (63)

\section{INTRODUCTION}

Carbon allotropy is considered to be one of the richest and most interesting. Due to its special physicochemical properties, carbon is able to crystallize into materials that have record characteristics: diamond is the hardest material in the world [1], graphene (ideal) has record electrical and thermal conductivity [2,3], some carbon materials have a record specific surface area [4]. The class of carbon nanotubes stands apart, which has a considerable potential for practical application because of an interesting combination of properties, as well as the presence of a large potential in the production of nanotubes with programmable properties $[5,6]$. 
In this paper, we describe a method for the production of graphene microtubes-in some way another allotropic modification of carbon materials. A graphene microtubule is a carbon tube consisting of grains of multilayer graphene held together by van der Waals forces, which can not be attributed either to graphene (mainly due to geometry) or to nanotubes (due to the polycrystalline multilayer structure). The basis of the work was the principle of making graphene the shape of the substrate, on which it is condensed from the gas phase in a CVD reactor [7]. This method of obtaining graphene microtubules differs from the method proposed, for example, in [8] or [9].

\section{MATERIALS AND METHODS}

Graphene microtubes were prepared by precipitating graphene into copper fiber (copper wire) in a CVD reactor. Then the copper substrate was poured into the solution of ferric chloride and as a result of this a graphene microtubule remained in the solution.

The substrate was copper wire type "M2" with a copper content of $99.7 \%$ with a diameter of 200 microns.

The preparation of wires for CVD experiments included the following stages:

- removal of lacquer residues in concentrated $(95 \%)$ warm $\left(50^{\circ} \mathrm{C}\right)$ sulfuric acid;

- washing in distilled water;

- ultrasonic cleaning first in acetone, then in isopropyl alcohol (for 15 minutes);

- electrochemical polishing of foils in a three-electrode cell on a potentiostategalvanostate P-30J of Elins in $87 \%$

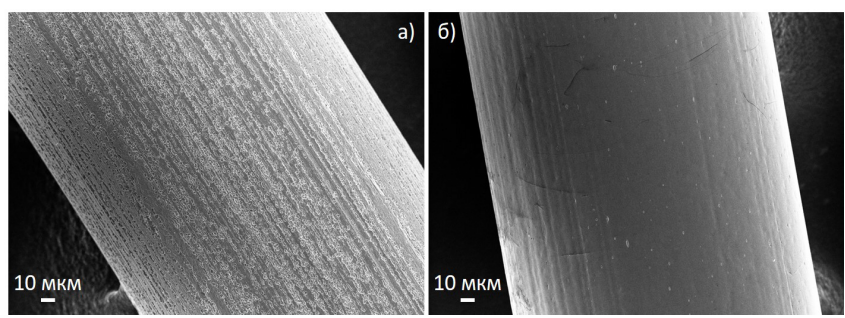

Fig. 1. Copper fiber before (a) and after (b) electropolishing.

orthophosphoric acid at room temperature with copper electrodes in potentiostatic mode with a constant voltage of 1400 $\mathrm{mV}$ for $8 \mathrm{~min}$;

- ultrasonic cleaning first in acetone, then in isopropyl alcohol (for 15 minutes).

In Fig. 1 shows the copper fiber before and after electropolishing. It can be seen that after polishing the surface became smoother, the surface roughness significantly decreased, which positively affects the continuity of the graphene coating.

Graphene was synthesized in the planarGROW-2S CVD reactor (Fig. 2) from planarTECH LCC (South Korea). The operation of the reactor (including auxiliary equipment) is fully automated and controlled using the SCADA shell made in the LabVIEW environment.

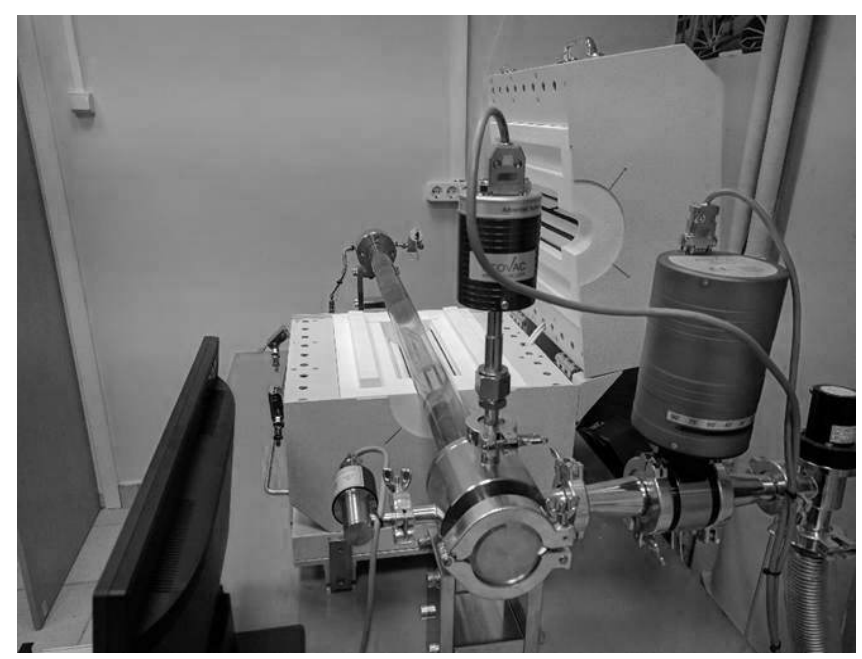

Fig. 2. CVD reactor 
Table 1. Stage 1. Annealing of the stand (preset parameters).

\begin{tabular}{|c|c|c|c|c|c|c|c|}
\hline Step & $\mathrm{T}^{\circ} \mathrm{C}$ & $\mathrm{t}, \mathrm{min}$ & $\begin{array}{c}\mathrm{Ar}, \\
\mathrm{sccm}\end{array}$ & $\begin{array}{c}\mathrm{H}_{2}, \\
\text { sccm }\end{array}$ & $\begin{array}{c}\mathrm{CH}_{4}, \\
\mathrm{sccm}\end{array}$ & $\begin{array}{c}\mathrm{NH}_{3}, \\
\mathrm{sccm}\end{array}$ & $\begin{array}{c}\text { P total. } \\
\text { Torr }\end{array}$ \\
\hline 1 & 1000 & 30 & 800 & 50 & 0 & - & 0 \\
\hline 2 & 1000 & 20 & 800 & 50 & 0 & - & 0 \\
\hline 3 & 0 & 60 & 800 & 50 & 0 & - & 0 \\
\hline
\end{tabular}

Inside the reactor (a quartz tube with an internal diameter of $4 \mathrm{~cm}$ and a length of about one meter), the copper fibers were located inside the cylinder of the quartz stand (or in the casing on the stand, see below).

The operating mode of the reactor for obtaining graphene on copper foils was selected experimentally. It consisted of two stages. At the first stage, reflected in Table 1, the stand was annealed. The second stage, which consisted in the synthesis of graphene, is reflected in Table 2.

The cooling of the reactor immediately after the growth of graphene took place naturally with the reactor cover closed: up to $700^{\circ} \mathrm{C}$ on average in $30-40$ minutes, then the furnace moved along the guide rails and the tube cooled to room temperature due to active air convection.

It should be noted that after the CVD experiments, the copper fibers looked like they looked like before, except that after the experiments the fibers acquired a natural copper luster due to the reduction of the oxide film.

The obtained samples were characterized by a scanning electron microscope (SEM, Carl

Stage 2. Growth of graphene (preset parameters).

\begin{tabular}{|l|l|l|c|c|c|c|c|}
\hline Step & \multicolumn{1}{|c|}{${ }^{\circ} \mathrm{C}$} & $\mathrm{t}, \mathrm{min}$ & $\begin{array}{c}\mathrm{Ar}, \\
\mathrm{sccm}\end{array}$ & $\begin{array}{c}\mathrm{H}_{2}, \\
\mathrm{sccm}\end{array}$ & $\begin{array}{c}\mathrm{CH}_{4}, \\
\mathrm{sccm}\end{array}$ & $\begin{array}{c}\mathrm{NH}_{3}, \\
\mathrm{sccm}\end{array}$ & $\begin{array}{c}\mathrm{P} \text { total. } \\
\text { Torr }\end{array}$ \\
\hline 1 & 0 & 5 & 100 & 30 & 50 & - & 7 \\
\hline 2 & 1005 & 60 & 200 & 30 & 0 & - & 7 \\
\hline 3 & 1005 & 30 & 200 & 30 & 0 & - & 7 \\
\hline 4 & 1005 & 20 & 200 & 30 & 20 & - & 7 \\
\hline 5 & 20 & 30 & 200 & 30 & 0 & - & 7 \\
\hline
\end{tabular}

Zeiss Supra 40-30-87, Germany), a Raman spectrometer (CD, NT-MDT INTEGRA Spectra, Russia, wavelength $472.9 \mathrm{~nm}$ ). X-ray spectra were obtained with the LYRA3 TESCAN scanning electron microscope equipped with an $\mathrm{x}$-ray detector from Oxford Instruments. Image processing and spectrum construction were carried out using Wolfram Mathematica and ImageJ software.

\section{RESULTS AND DISCUSSION}

\subsection{IMPURITY STRUCTURES}

The first CVD experiments with the production of graphene on a wire revealed a number of features compared to the production of graphene on a flat surface. In Fig. 3 shows the surface of the copper fiber after the CVD experiment. It can be seen that it is all strewn with light impurities (against the background of the copper fiber surface and grains of graphene) by impurity structures that interfere with the formation of a continuous graphene coating. X-ray spectra of similar structures showed an increased content of silicon and oxygen in comparison with the rest of the surface (Fig. 4). From this it can be concluded that the impurity structures are a phase containing silicon oxides.

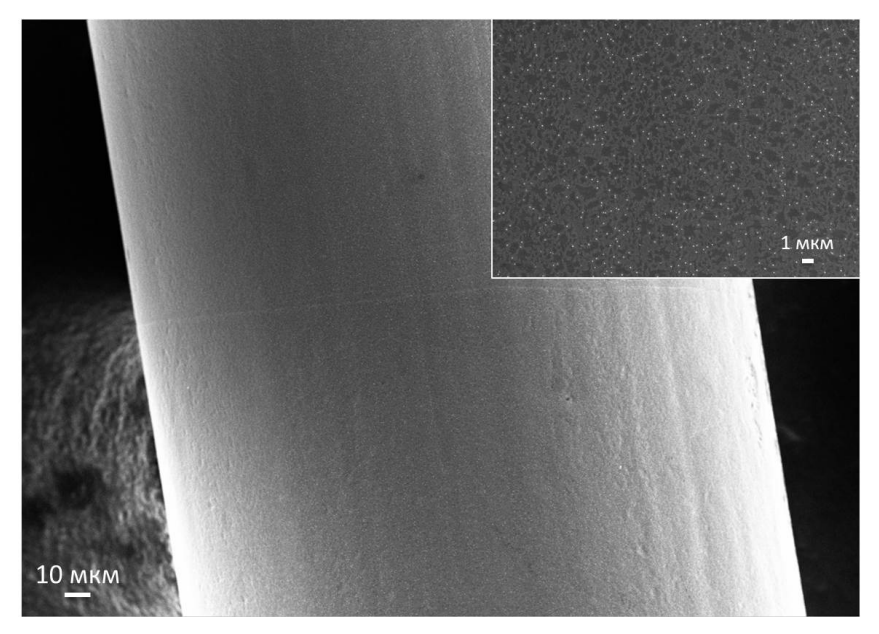

Fig. 3. The surface of the copper fiber after the CVD experiment without the use of a copper casing. 


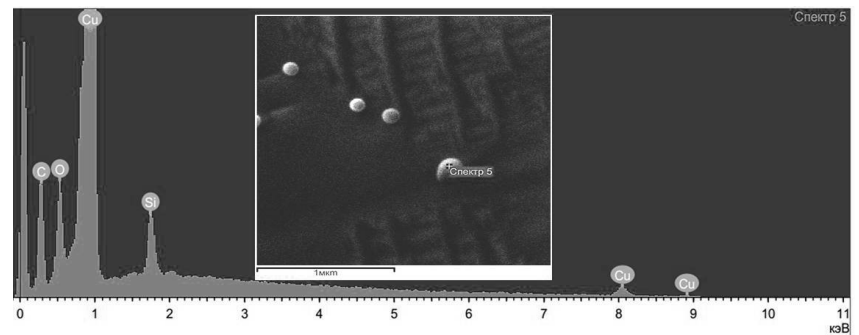

Fig. 4. X-ray spectrum of the impurity structure on the surface of the copper fiber that was formed during the CVD experiment.

Impurity particles of similar morphology on copper fibers were observed in [10] and [11].

Apparently, the quartz tube of the reactor and the quartz substrate for the substrates are the main source of silicon in the core of the CVD reactor: at high temperature and in vacuum, silicon (or silicon oxides) sublimates and can condense on copper substrates. A large number of impurity structures can be explained by the fact that the nucleation of these particles containing silicon occurs to a large extent on the grain boundaries, which, due to the geometry, are very much on the fibers. This is also confirmed by SEM micrographs, which show an increased concentration of impurity particles near grain boundaries (not shown here).

\subsection{Growth of graphene on copper fiber}

An attempt was made to reduce the content of impurity structures on the surface of copper fibers and to improve the morphology of the graphene coating by using a copper casing, inside which was placed copper fiber. The results were encouraging: on a fiber placed in a copper casing (graphene growth actually occurs in copper vapor), the graphene coating looks quite solid with an average characteristic island size of about 10 microns. At the same time, impurity structures were practically not observed. The
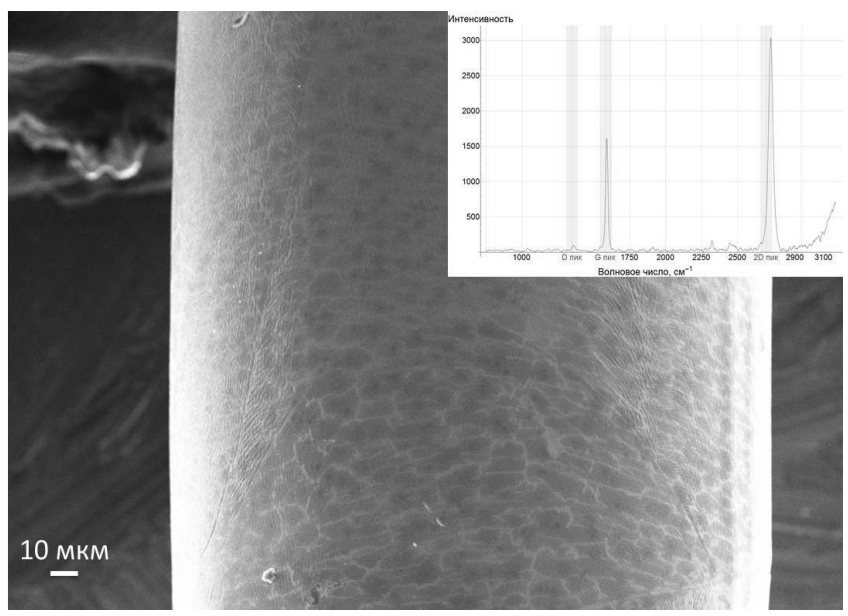

Fig. 5. The surface of the copper fiber after the CVD experiment using a copper casing. On the inset, the Raman spectrum of a graphene coating.

Raman spectra also show that the graphene coating on the wire has a poorly defective, multilayered character (Fig. 5).

To verify the continuity of the graphene coating obtained on the wire, an attempt was made to obtain a graphene microtubule by etching out the substrate (copper) on which it was obtained (Fig. 6).

For etching copper, a $1 \mathrm{M}$ solution of ferric chloride in water was used. Within a few hours the copper core was dissolved, exposing the graphene shell - a graphene microtubule. The resulting microtubule proved to be strong enough to not break down when moving in a liquid by disturbing it with vibrational motions. To seize it, however, tweezers fail because of its destruction. In part due to this, it was virtually impossible to characterize the received tube. However,

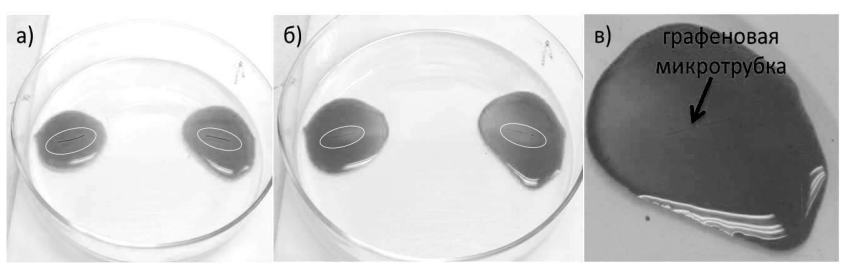

Fig. 6. The process of etching copper fiber in FeCl3 solution: a) beginning of etching, b) after 40 min, c) after 2 hours. 
with some confidence, it can be argued that the Raman spectrum of such a microtubule is similar to that obtained from a copper fiber on which the graphene coating was prior to etching.

\section{CONCLUSION}

The paper shows the potential to extend the class of flat graphenes (obtained on flat surfaces) to graphenes of a rather arbitrary shape, depending on the geometry of the substrate on which graphene is grown.

Using as a substrate a copper fiber (cylindrical surface) with a diameter of 200 $\mu \mathrm{m}$, it was possible to obtain a graphene microtubule in the work, which retained its integrity after dissolving the carrier substrate.

To get rid of impurity structures (presumably silicon oxides) on the surface of copper fiber and improve the quality and continuity of graphene, a copper casing was used during its growth. In addition, graphene islands on fibers obtained with a copper casing have an increased average characteristic size compared to experiments without a casing, which reduces the number of grain boundaries and improves the uniformity of the coating.

The work was carried out within the framework of RFBR grant 16-03-00132.

\section{REFERENCES}

1. Brookes CA, Brookes EJ. Diamond in perspective: a review of mechanical properties of natural dia-mond. Diamond and Related Materials, 1991, 1(1):13-17.

2. Geim AK, Novoselov KS. The rise of graphene. Nature Mater., 2007, 6(3):183-191.
3. Soldano C, Mahmood A, Dujardin E. Production, properties and potential of graphene. Carbon, 2010, 48(8):2127-2150.

4. Fei Xu, Zhiwei Tang, Siqi Huang, Luyi Chen, Yeru Liang, Weicong Mai, Hui Zhong, Ruowen Fu, Dingcai Wu. Facile synthesis of ultrahigh-surface-area hollow carbon nanospheres for enhanced adsorption and energy storage. Nature Communications, 2015, 6:7863.

5. Trojanowicz M. Analytical applications of carbon nanotubes: a review. TrAC Trends in Analytical Chemistry, 2006, 25(5):480-489.

6. Jing Liu, Junzhe Lu, Xiang Lin, Yuchao Tang, Yanan Liu, Ting Wang, Hengjiang Zhu. The electronic properties of chiral carbon nanotubes. Computational Materials Science, 2017, 129:290-294.

7. Zhang Yi, Zhang Luyao, Zhou Chongwu. Review of chemical vapor deposition of graphene and related applications. Acc. Chem. Res., 2013, 46(10):2329-2339.

8. Guo W, Qiu J, Liu J, Liu H. Graphene microfiber as a scaffold for regulation of neural stem cells differentiation. Scientific Reports, 2017, 7(1):5678.

9. Yao BC, Wu Y, Zhang AQ, Rao YJ, Wang ZG, Cheng Y, Gong Y, Zhang WL, Chen YF, Chiang KS. Graphene enhanced evanescent field in microfiber multi-mode interferometer for highly sensitive gas sensing. Optics Express, 2014, 22(23):28154-28162.

10. Ruiz I, Wang W, George A, Ozkan M. Silicon oxide contamination of grapheme sheets synthesized on copper substrates via chemical vapor deposition. Advanced 
Science, Engineering and Medicine, 2014, 6(10):1-6.

11. Çelik Y, Escoffier W, Yang M, Flahaut E, Suvaci E. Relationship between heating atmosphere and copper foil impurities during graphene growth via low pressure chemical vapor deposition. Carbon, 2016, 109:529-541. 